Research Article; Received: July 23, 2020; Accepted: May 10, 2021

\title{
DISCRETIZATION AND CHAOS CONTROL IN A FRACTIONAL ORDER PREDATOR-PREY HARVESTING MODEL
}

\author{
George M. S. AMIRTHARAJ ${ }^{1}$, Janagaraj RAJENDRAN ${ }^{2}$, Vignesh DHAKSHINAMOORTHY ${ }^{1}$ \\ ${ }^{1}$ PG \& Research Department of Mathematics, Sacred Heart College (Autonomous), \\ Tirupattur-635601, Tamil Nadu, INDIA \\ ${ }^{2}$ Department of Mathematics, Faculty of Engineering, Karpagam Academy of Higher Education, \\ Coimbatore-641021, Tamil Nadu, INDIA
}

\begin{abstract}
The study of interaction between predator and prey species is one of the important subjects in mathematical biology. Optimal strategy control plays a vital role in preserving animals from extinction. Harvesting of species is a vital issue for the conservation biologists. In this work, we investigate the bifurcation and chaos control of the two species interaction model of fractional order in discrete time with harvesting of both prey and predator species. Existence results and the stability conditions of the system are analyzed using the fixed points and jacobian matrix. The chaotic behavior of the system is discussed with bifurcation diagrams. Linear control and hybrid control methods are used in controlling the chaos of the system. Numerical experiments with different phase portraits are simulated for the better understanding of the qualitative behavior of the considered model.
\end{abstract}

\section{INTRODUCTION}

Modelling of real life phenomena by fractional order equations is more realistic and follows the laws of nature very well. Fractional calculus is used in modelling of physical and chemical phenomena like diffusion waves, nonlinear oscillations involved in earthquakes, hydrologic models, blood vessel models and various other interdisciplinary fields. In construction of biological models, fractional calculus relates the memory effects of biological populations very well rather than ordinary

2020 Mathematics Subject Classification. Primary 34A08, 37N25, 39A28; Secondary 39A30, 92D25.

Keywords. Population dynamics, fractional order, discrete, Neimark-Sacker bifurcation, Flip bifurcations.

『agmshc@gmail.com-Corresponding author; jangarajtk@gmail.com; dvignesh260@gmail.com

(D) 0000-0003-2004-3537; 0000-0002-9811-078X; 0000-0002-9942-4035.

Communications Faculty of Sciences University of Ankara Series A1 (C)2021 Ankara University 
integer order calculus. Recently, models of species interaction and biological populations are developed using fractional calculus with discrete time [2, 21, 22, 24, 25.

Mathematical models of the species by Lotka in 1925 and Volterra in 1926 were the first models on the interactions involving multi species [11. Later several models on interacting species were developed by Robert May in 1972, Holling and Tanner in 1975 and many other researchers proving the necessity in studying mathematical ecology [15, 26. Biological populations with non overlapping generations are modeled with difference equations and some of the discrete time models are studied by [8, 9, 12, 13, 14.

The population dynamics gives an accurate and deep understanding of the factors threatening the existence of the species in ecosystem. Apart from the natural forces like drought and natural calamities there are some artificial factors that has human involvement such as hunting, human habitat and harvesting also paves way for the extinction of the species. Continuous harvesting results in unstability of ecosystem with loss in biodiversity. Thus, it is necessary to bring forth some conservation policy for optimal harvesting of species. Myerscough et al [20] reported the effects of predator harvesting on ecosystem, harvesting and its effects in aquasystem was studied in [16], 19] studied the prey-predator system with constant harvesting policy and the fractional order model of quadratic harvesting of scavenger was studied in 23 .

The paper is structured with discretization of fractional order system in section 2 followed with analysis of stability condition in Section 3. The bifurcation analysis and chaos control in section 4 and 5 respectively. Section 6 provides some numerical examples with simulations.

\section{Fractional Order System with Discretization}

In the recent decades, Fractional order has emerged as one of the significant interdisciplinary subjects in physical \& biological sciences and Engineering [4, 3], 1]. In this work, the biological system with harvesting of predator and prey species are considered. The non-dimensional form of system is

$$
\begin{aligned}
& \frac{d x}{d t}=s x(1-x)-\beta x y-c x \\
& \frac{d y}{d t}=-w y+\eta x y-f y
\end{aligned}
$$

In system (1), the prey and predator populations are represented by $x(t)$ and $y(t)$. All the system parameters $s, \beta, c, w, \eta, f$ take positive real values that stand for growth rate of prey, interaction rate, harvesting effort of prey, mortality rate of predator, conversion rate of prey and harvesting effort of predator respectively.

Generalization of (1) to arbitrary order yield

$$
\begin{aligned}
& D_{t}^{v}(x)=s x(1-x)-\beta x y-c x \\
& D_{t}^{v}(y)=-w y+\eta x y-f y
\end{aligned}
$$


with $x(0)=x_{0}$ and $y(0)=y_{0}$, where $v \in(0,1)$ is non integer order and fractional order caputo derivative is ${ }_{a} D_{t}^{v} f(t)=\frac{1}{\Gamma(1-v)} \int_{a}^{t} \frac{f^{(n)}(\tau)}{(t-\tau)^{v-n+1}} d \tau$, for $n-1<v<n[5]$.

2.1. Discretization Process. The discretization of the system (2) with initial point $x(0)=x_{0}$ and $y(0)=y_{0}$ is carried out using piecewise constant arguments method [2, 7]. The fractional order predator prey system with harvesting at discrete time is

$$
\begin{aligned}
& x(t+1)=x(t)+\frac{\rho^{\nu}}{\Gamma(1+\nu)}[s x(t)(1-x(t))-\beta x(t) y(t)-c x(t)] \\
& y(t+1)=y(t)+\frac{\rho^{\nu}}{\Gamma(1+\nu)}[-w y(t)+\eta x(t) y(t)-f y(t)]
\end{aligned}
$$

where $v \in(0,1]$ and $\rho>0$ is step size.

2.2. Existence and Uniqueness of the Solution. Let the region be defined by $\Theta \times(0, \mathfrak{T}]$ where

$$
\Theta=\left\{(x, y) \in \mathfrak{R}^{2}: \max (|x|,|y| \leq L)\right\}
$$

The existence results are established with method as in [17]. Consider a mapping $\mathcal{H}(C)=\left(\mathcal{H}_{1}(C), \mathcal{H}_{2}(C)\right)$ such that

$$
\begin{aligned}
& \mathcal{H}_{1}(C)=s x(1-x)-\beta x y-c x \\
& \mathcal{H}_{2}(C)=-w y+\eta x y-f y .
\end{aligned}
$$

Let $C, \bar{C} \in \Theta$. We have from (4) that

$$
\begin{aligned}
\|\mathcal{H}(C)-\mathcal{H}(\bar{C})\| & =\left|\mathcal{H}_{1}(C)-\mathcal{H}_{1}(\bar{C})\right|+\left|\mathcal{H}_{2}(C)-\mathcal{H}_{2}(\bar{C})\right| \\
& =|s x(1-x)-\beta x y-c x-s \bar{x}(1-\bar{x})+\beta \bar{x} \bar{y}+c \bar{x}| \\
& +|-w y+\eta x y-f y+w \bar{y}-\eta \bar{x} \bar{y}+f \bar{y}| \\
& =|s(x-\bar{x})-s(x-\bar{x})(x+\bar{x})-\beta(x y-\bar{x} \bar{y})-c(x-\bar{x})| \\
& +|\eta(x y-\bar{x} \bar{y})-w(y-\bar{y})-f(y-\bar{y})| \\
& \leq s|(x-\bar{x})|+2 s L|x-\bar{x}|+\beta L|(y-\bar{y})|+\beta L|(x-\bar{x})| \\
& +c|(x-\bar{x})|+\eta|(x-\bar{x})|+\eta(y-\bar{y})|+w|(y-\bar{y})|+f|(y-\bar{y}) \mid \\
& \leq[(1+2 L) s+(\beta+\eta) L+c]|x-\bar{x}|+[(\beta+\eta) L+w+f]|y-\bar{y}| \\
\|\mathcal{H}(C)-\mathcal{H}(\bar{C})\| & \leq \Omega\|C-\bar{C}\|
\end{aligned}
$$

and

$$
\Omega=\max \{(1+2 L) s+(\beta+\eta) L+c,(\beta+\eta) L+w+f\} .
$$

For $\Omega<1$, we obtain $C=\mathcal{H}(C)$ and hence $\mathcal{H}(C)$ is a contraction mapping.

Theorem 1. The sufficient condition for existence of unique solution of the fractional system $(2)$ in $\Theta \times(0, \mathfrak{T}]$ is

$$
\Omega=\max \{(1+2 L) s+(\beta+\eta) L+c,(\beta+\eta) L+w+f\}<1 .
$$




\section{Equilibrium points And Stability of System 3}

This section investigates the stability results of the (3) using Jury conditions.

3.1. Equilibrium Points and its Existence. The positive equilibrium points of system (3) are obtained by solving

$$
\begin{array}{r}
s x(1-x)-\beta x y-c x=0 \\
-w y+\eta x y-f y=0 .
\end{array}
$$

(1) $E S_{0}=(0,0)$

(2) $E S_{1}=\left(\frac{s-c}{s}, 0\right)$

(3) $\mathrm{ES}_{2}=\left(x^{*}, \frac{s-c}{\beta}-\frac{s}{\beta} x^{*}\right)$.

where $x^{*}=\frac{w+f}{\eta}$.

Theorem 2. The equilibrium points satisfy

(1) The trivial equilibrium point $E S_{0}$ always exists.

(2) The axial equilibrium steady state $E S_{1}$ exists if $s>c$

(3) The interior equilibrium point $E S_{2}$ exists if $\eta>\frac{s(w+f)}{s-c}$.

3.2. Stability Results of System (3). Jacobian matrices are formulated at the steady states and jury conditions are employed to investigate the stability of the system (3). At $(x, y)$, the Jacobian matrix is

$$
J(x, y)=\left[\begin{array}{cc}
1+Q[s(1-2 x)-\beta y-c] & -Q \beta x \\
Q \eta y & 1+Q[\eta x-w-f]
\end{array}\right]
$$

where $Q=\frac{\rho^{v}}{\Gamma(1+v)}$. Now the characteristic equation of $(6)$ is

$$
\Phi(m)=m^{2}-T m+D=0
$$

where $T=2+Q[s-c-w-f+(\eta-2 s) x-\beta y]$ is the trace of (6) and determinant of (6) is $D=1+Q[s-c-w-f-\beta y+x(\eta-2 s)]$ $+Q^{2}[(s-2 s x-c)(\eta x-w-f)+\beta y(w+f)]$.

Lemma 3. [18] Let $m_{1}, m_{2}$ satisfy $\Phi(m)=0$ and suppose that $\Phi(1)>0$. Then $\left(x^{*}, y^{*}\right)$ is

(1) stable if $\left|m_{1}\right|<1,\left|m_{2}\right|<1 \Leftrightarrow \Phi(-1)>0, \Phi(0)<1$.

(2) saddle point if $\left|m_{1}\right|<1,\left|m_{2}\right|>1$ (or $\left.\left|m_{1}\right|>1,\left|m_{2}\right|<1\right) \Leftrightarrow \Phi(-1)<0$.

(3) unstable if $\left|m_{1}\right|>1,\left|m_{2}\right|>1 \Leftrightarrow \Phi(-1)>0, \Phi(0)>1$.

(4) $\left|m_{1}\right|=-1,\left|m_{2}\right| \neq 1 \Leftrightarrow \Phi(-1)=0$.

(5) $m_{1}, m_{2}$ are complex and $\left|m_{1}\right|=\left|m_{2}\right| \Leftrightarrow T^{2}-4 D<0$ and $\Phi(0)=1$.

Theorem 4. The equilibrium point $E S_{0}$ is

(a) unstable for $\left|m_{2}\right|>1$ i.e. $\rho>\left[\frac{2 \Gamma(1+v)}{w+f}\right]^{\frac{1}{v}}$.

(b) saddle point for $\left|m_{2}\right|<1$, i.e. $0<\rho<\left[\frac{2 \Gamma(1+v)}{w+f}\right]^{\frac{1}{v}}$. 
(c) non-hyperbolic for $\rho=\left[\frac{2 \Gamma(1+v)}{c}\right]^{\frac{1}{v}}$.

Proof. At $E S_{0},(6)$ becomes

$$
J_{E S_{0}}=J(0,0)=\left[\begin{array}{cc}
1+Q(s-c) & 0 \\
0 & 1-Q(w+f)
\end{array}\right]
$$

whose eigenvalues are $m_{1}=1+Q(s-c)$ and $m_{2}=1-Q(w+f)$. Since $\frac{\rho^{v}}{\Gamma(1+v)}>0$ for $0<v \leq 1$.

(a) It is obvious that $\left|m_{2}\right|>1$. Then $E S_{0}$ is source if $|1-Q(w+f)|>1$ which yields $\rho>\left[\frac{2 \Gamma(1+v)}{w+f}\right]^{\frac{1}{v}}$.

(b) $E S_{0}$ is saddle point if $|1-Q(w+f)|<1$, i.e. $0<\rho<\left[\frac{2 \Gamma(1+v)}{w+f}\right]^{\frac{1}{v}}$.

(c) This result is a consequence of (I) and (II).

Theorem 5. The axial equilibrium point $E S_{1}$ is

(a) stable for $c<s<\frac{\eta c}{\eta-w-f}$ and $\rho<\min \left\{\left[\frac{2 \Gamma(1+v)}{s-c}\right]^{\frac{1}{v}},\left[\frac{2 \Gamma(1+v)}{s(w+f)-\eta(s-c)}\right]^{\frac{1}{v}}\right\}$.

(b) unstable for $\frac{\eta c}{\eta-w-f}<s<c$ and $\rho>\max \left\{\left[\frac{2 \Gamma(1+v)}{s-c}\right]^{\frac{1}{v}},\left[\frac{2 \Gamma(1+v)}{s(w+f)-\eta(s-c)}\right]^{\frac{1}{v}}\right\}$.

(c) saddle for $s>\max \left\{\frac{\eta c}{\eta-w-f}, c\right\}$ and $\left[\frac{2 \Gamma(1+v)}{s(w+f)-\eta(s-c)}\right]^{\frac{1}{v}}<\rho<\left\{\left[\frac{2 \Gamma(1+v)}{s-c}\right]^{\frac{1}{v}}\right\}$.

(d) non-hyperbolic for $s=c($ or $) s=\left\{\frac{\eta c}{\eta-w-f}\right\}$ and $\rho=\left[\frac{2 \Gamma(1+v)}{s(w+f)-\eta(s-c)}\right]^{\frac{1}{v}}$ (or) $\rho=\left\{\left[\frac{2 \Gamma(1+v)}{s-c}\right]^{\frac{1}{v}}\right\}$.

Proof. For $E S_{1}$, Jacobian matrix is

$$
J_{E S_{1}}=J\left(\frac{s-c}{s}, 0\right)=\left[\begin{array}{cc}
1+Q(s-c) & -Q \frac{\beta(s-c)}{s} \\
0 & 1-Q\left[w+f-\frac{\eta(s-c)}{s}\right]
\end{array}\right]
$$

whose eigen values are $m_{1}=1-Q(s-c)$ and $m_{2}=1-Q\left[w+f-\frac{\eta(s-c)}{s}\right]$. Since $\frac{\rho^{v}}{\Gamma(1+v)}>0$ for $0<\alpha \leq 1$.

(a) $E S_{1}$ is stable if $|1-Q(s-c)|<1$ and $\left|1-Q\left[w+f-\frac{\eta(s-c)}{s}\right]\right|<1$ which yields

$$
c<s<\frac{\eta c}{\eta-w-f} \text { and } \rho<\min \left\{\left[\frac{2 \Gamma(1+v)}{s-c}\right]^{\frac{1}{v}},\left[\frac{2 \Gamma(1+v)}{s(w+f)-\eta(s-c)}\right]^{\frac{1}{v}}\right\} \text {. }
$$


(b) $E S_{1}$ is unstable if $|1-Q(s-c)|>1$ and $\left|1-Q\left[w+f-\frac{\eta(s-c)}{s}\right]\right|>1$, i.e.

$$
\frac{\eta c}{\eta-w-f}<s<c \text { and } \rho>\max \left\{\left[\frac{2 \Gamma(1+v)}{s-c}\right]^{\frac{1}{v}},\left[\frac{2 \Gamma(1+v)}{s(w+f)-\eta(s-c)}\right]^{\frac{1}{v}}\right\}
$$

(c) $E S_{1}$ is Saddle if $|1-Q(s-c)|<1$ and $\left|1-Q\left[w+f-\frac{\eta(s-c)}{s}\right]\right|>1$, i.e.

$$
s>\max \left\{\frac{\eta c}{\eta-w-f}, c\right\} \text { and }\left[\frac{2 \Gamma(1+v)}{s(w+f)-\eta(s-c)}\right]^{\frac{1}{v}}<\rho<\left\{\left[\frac{2 \Gamma(1+v)}{s-c}\right]^{\frac{1}{v}}\right\}
$$

(d) The proof follows from result (a) and (b).

At $E S_{2},(6)$ becomes

$$
J_{E S_{2}}=\left[\begin{array}{cc}
1+Q a_{11} & -Q a_{12} \\
Q a_{21} & 1
\end{array}\right]
$$

The characteristic equation of $J_{\mathrm{ES}_{2}}$ is $\Phi(m)=m^{2}-T m+D=0$, with $T=2+Q a_{11}$ and $D=1+Q a_{11}+Q^{2} a_{12} a_{21}$, where $Q=\frac{\rho^{v}}{\Gamma(1+v)}, a_{11}=-\frac{s(w+f)}{\eta}, a_{12}=\frac{\beta(w+f)}{\eta}$ and $a_{21}=\frac{\eta(s-c)-s(w+f)}{\beta}$. Eigen values are

$$
m_{1,2}=1+\frac{Q M}{2} \pm \frac{Q}{2} \sqrt{M^{2}-4 N},
$$

here $M=a_{11}$ and $N=a_{12} a_{21}$.

Theorem 6. The interior equilibrium point $E S_{2}$ is a

(a) sink if one of the following conditions are satisfied:

(i) $\mathfrak{S}^{*}<0$ and $\rho<\rho_{3}$,

(ii) $\mathfrak{S}^{*} \geq 0$ and $\rho<\rho_{2}$,

(b) source if one of the following conditions are satisfied:

(i) $\mathfrak{S}^{*}<0$ and $\rho>\rho_{3}$,

(ii) $\mathfrak{S}^{*} \geq 0$ and $\rho>\rho_{1}$,

(c) saddle, if

(i) $\mathfrak{S}^{*} \geq 0$ and $\rho_{2}<\rho<\rho_{1}$,

(d) non-hyperbolic, if one of the following conditions are satisfied:

(i) $\mathfrak{S}^{*}<0$ and $\rho=\rho_{3}$.

(ii) $\mathfrak{S}^{*}>0$ and $\rho=\rho_{1}$ or $\rho=\rho_{2}$,

$$
\begin{gathered}
\mathfrak{S}^{*}=\left(M^{2}-4 N\right) \text { and } \rho_{1}=\left\{\Gamma(1+v)\left[\frac{-M+\sqrt{M^{2}-4 N}}{N}\right]\right\}^{\frac{1}{v}}, \\
\rho_{2}=\left\{\Gamma(1+v)\left[\frac{-M-\sqrt{M^{2}-4 N}}{N}\right]\right\}^{\frac{1}{v}}, \rho_{3}=\left\{\left[\frac{-\Gamma(1+v) M}{N}\right]\right\}^{\frac{1}{v}}
\end{gathered}
$$

\section{Bifurcation Analysis of (3)}

The existence of bifurcation is investigated in this section. 
4.1. Periodic Doubling Bifurcation. The parameter for analyzing existence of bifurcation is chosen as $\rho$. The equilibrium point $E S_{2}$ is said to undergo periodic doubling bifurcation if one of the eigenvalue is -1 and other shall not be 1 (or) -1 [10].

The quadratic equation obtained from $(8)$ is

$$
\Phi(m)=m^{2}-(2+Q M) m+\left(1+Q M+Q^{2} N\right) .
$$

By Theorem (6), if $S^{*} \geq 0$ and $\rho=\left[\frac{2 \Gamma(1+v)}{s-c}\right]^{\frac{1}{v}}$, the eigen values are

$$
m_{1}=-1, m_{2}=1-\frac{2(w+f)}{s-c}+\frac{2 \eta}{s} .
$$

Theorem 7. The periodic doubling bifurcation occurs causing instability to ES $S_{1}$ when $S^{*}=\left(Q(s-c)-Q\left(w+f-\frac{\eta(s-c)}{s}\right)\right)^{2} \geq 0$ and $\rho=\left[\frac{2 \Gamma(1+v)}{s-c}\right]^{\frac{1}{v}}$, and

$$
m_{1}=-1, m_{2}=1-\frac{2(w+f)}{s-c}+\frac{2 \eta}{s} \neq \pm 1 .
$$

4.2. Neimark Sacker Bifurcation. Let $\rho$ be the bifurcation parameter considered to analyzes Neimark-Sacker bifurcation. The occurance of this bifurcation is ensured when the eigenvalues at steady state $E S_{2}$ are complex conjugate with modulus equal to 1 [10]. The quadratic equation obtained from (8) is

$$
\Phi(m)=m^{2}-(2+Q M) m+\left(1+Q M+Q^{2} N\right) .
$$

From Theorem (6), if $S^{*}<0$ and $\rho=\rho_{3}$, then

$$
m_{1,2}=1-\frac{M^{2}}{2 N} \pm i \frac{M}{2 N} \sqrt{4 N-M^{2}} .
$$

are the corresponding eigen values.

Theorem 8. The Neimark-Sacker bifurcation of system (3) occurs when $S^{*}<0$ and $\rho=\rho_{3}$, and

$$
\left|m_{1,2}\right|=\left|1-\frac{M^{2}}{2 N} \pm i \frac{M}{2 N} \sqrt{4 N-M^{2}}\right|=1 \text {. }
$$

\section{Control Strategies}

The system with linear feedback controller [6] is

$$
\begin{aligned}
& x(t+1)=x(t)+\frac{\rho^{v}}{\Gamma(1+v)}[s x(t)(1-x(t))-\beta x(t) y(t)-c x(t)]+R(t) \\
& y(t+1)=y(t)+\frac{\rho^{v}}{\Gamma(1+v)}[-w y(t)+\eta x(t) y(t)-f y(t)]
\end{aligned}
$$

where feedback control is $R(t)=-r_{1}\left(x(t)-\frac{s-c}{s}\right)-r_{2} y(t)$ with $r_{1}, r_{2}$ being feedback gains. The Jacobian of system $(9)$ at $\left(\frac{s-c}{s}, 0\right)$ is 


$$
J_{1}\left(\frac{s-c}{s}, 0\right)=\left[\begin{array}{cc}
1-Q A-r_{1} & -\frac{Q \beta A}{s}-r_{2} \\
0 & 1-Q B
\end{array}\right]
$$

Here $A=(s-c)$ and $B=\left[w+f-\frac{\eta(s-c)}{s}\right]$. The corresponding characteristic equation of $J_{1}\left(\frac{s-c}{s}, 0\right)$ is

$$
m^{2}-\left(2-Q(A+B)-r_{1}\right) m+\left(Q^{2} A B-Q\left(A+B+B r_{1}\right)+1-r_{1}\right)=0 .
$$

If $m_{1}, m_{2}$ are the eigenvalues of 11 , then and

$$
m_{1,2}=\frac{\left(2-Q(A+B)-r_{1}\right) \pm \sqrt{\left(2-Q(A+B)-r_{1}\right)^{2}-4\left(Q^{2} A B-Q\left(A+B-B r_{1}\right)+1-r_{1}\right)}}{2}
$$

$$
m_{1} m_{2}=1-r_{1}-Q\left(A+B-B r_{1}\right)+Q^{2} A B
$$

The equations $m_{1}= \pm 1$ and $m_{1} m_{2}=1$ ensure that absolute values of the eigen values are less than 1 . Suppose $m_{1} m_{2}=1$, then $(12)$ becomes

$$
l_{1}: Q^{2} A B-Q(A+B)=r_{1}-Q B r_{1}
$$

Suppose that $m_{1}=1$ or $m_{1}=-1$, then equation (11) yields

$$
\begin{aligned}
& l_{2}:-Q A=r_{1} \\
& l_{3}: Q^{2} A B-2 Q(A+B)+4=2 r_{1}-Q B r_{1}
\end{aligned}
$$

The triangular region with lines $l_{1}, l_{2}$ and $l_{3}$ contains the eigenvalues.

Next, the system with hybrid controlled strategy is given by

$$
\begin{aligned}
& x(t+1)=\alpha x(t)+\frac{\alpha \rho^{\nu}}{\Gamma(1+\nu)}[s x(t)(1-x(t))-\beta x(t) y(t)-c x(t)]+(1-\alpha) x(t) \\
& y(t+1)=\alpha y(t)+\frac{\alpha \rho^{\nu}}{\Gamma(1+\nu)}[-w y(t)+\eta x(t) y(t)-f y(t)]+(1-\alpha) x(t)
\end{aligned}
$$

where $0<\alpha<1$. Parameter perturbation and feedback control are combined in (13) as control strategy and appropriate choice of $\alpha$ results in partial or completely elimination of Neimark sacker bifurcation. Jacobian of 13 at $E S_{2}$ is

$$
J_{2}\left(E S_{2}\right)=\left[\begin{array}{cc}
1+\alpha A a_{11} & -\alpha A a_{12} \\
\alpha A a_{21} & 1
\end{array}\right] .
$$

where $A, a_{11}, a_{12}, a_{21}$ are given in (8). The presence of the roots of the (14) in the unit disk ensure the asymptotic stability of $E S_{2}$. 


\section{NumERICAL EXPERIMENTS}

This section illustrates the results obtained above with suitable examples.

Example 9. Let $\nu=0.85, s=2.3, \beta=0.8, c=0.05, w=0.3, \eta=0.81, f=0.5$ and $0.75 \leq \rho \leq 1.31$ of system (3) and $x(0)=0.55, y(0)=0.45$. We obtain $E S_{1}=\left(x^{*}, y^{*}\right)=(0.9782,0)$. Eigen values are $m_{1}=-1$ and $m_{2}=0.9932 \neq 1$. The critical point of periodic doubling bifurcation given in Theorem (7) is $\rho=0.8151$. Figure 1(a), 1(b) show flip bifurcation diagrams in $(\rho, x)$ and lyapunov exponent. The periodic windows of the corresponding bifurcation diagrams are represented in $1(c), 1(d), 1(e)$ and $1(f)$ respectively.
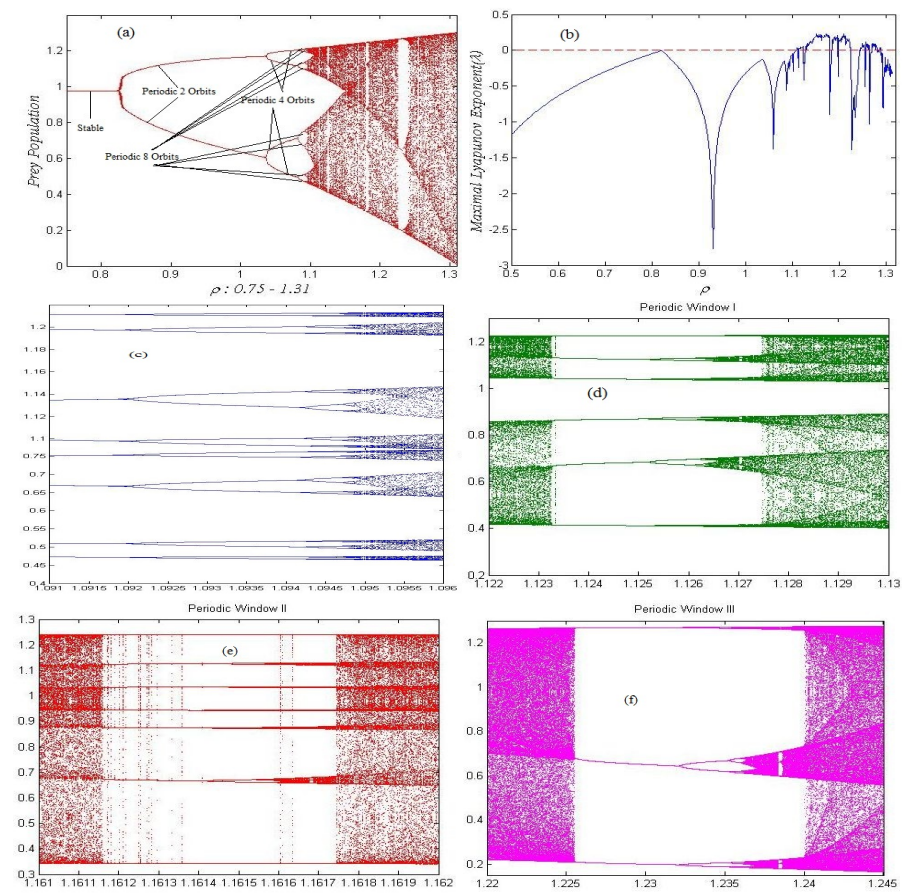

Figure 1. Flip bifurcation diagram in $(\rho, x)$ plane and Maximum Lyapunov exponents of the system (3) with different periodic windows

Example 10. Let $\nu=0.85, \rho=0.82, s=2.3, \beta=0.8, c=0.05, w=0.3, \eta=$ $0.81, f=0.5$ and $x(0)=0.55, y(0)=0.45$ for the system (3), periodic doubling bifurcation occurs as $\rho$ varies in $\rho \in[0.75,1.31]$. Moreover, Figure 2(a) displays the time plots for both prey and predator populations at $\rho=0.82$. 

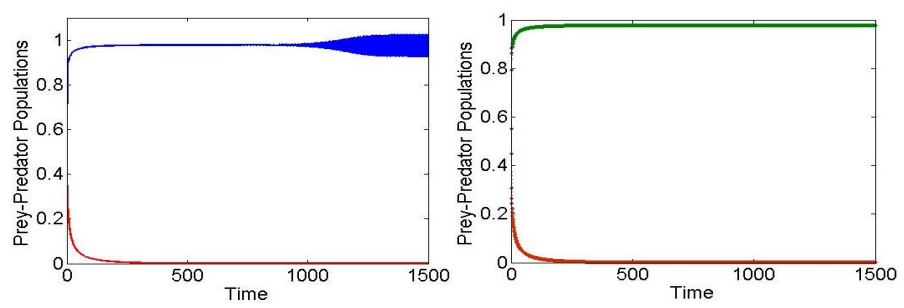

Figure 2. Time series for the system 3 and 15

The controlled system (9) for above values takes the form

$$
\begin{aligned}
& x(t+1)=x(t)+\frac{\rho^{v}}{\Gamma(1+v)}[s x(t)(1-x(t))-\beta x(t) y(t)-c x(t)]+R(t) \\
& y(t+1)=y(t)+\frac{\rho^{v}}{\Gamma(1+v)}[-w y(t)+\eta x(t) y(t)-f y(t)]
\end{aligned}
$$

where $R(t)=-r_{1}\left(x(t)-\frac{s-c}{s}\right)-r_{2} y(t)$ and $r_{1}=-0.07$ and $r_{2}=0.075$. The plots for the system (15) with control terms are provided in Figure 2(b). It is clear that the equilibrium $E S_{1}$ is stable. The time plots at different $\rho$ values are displayed in Figure 3 and Figure 4.
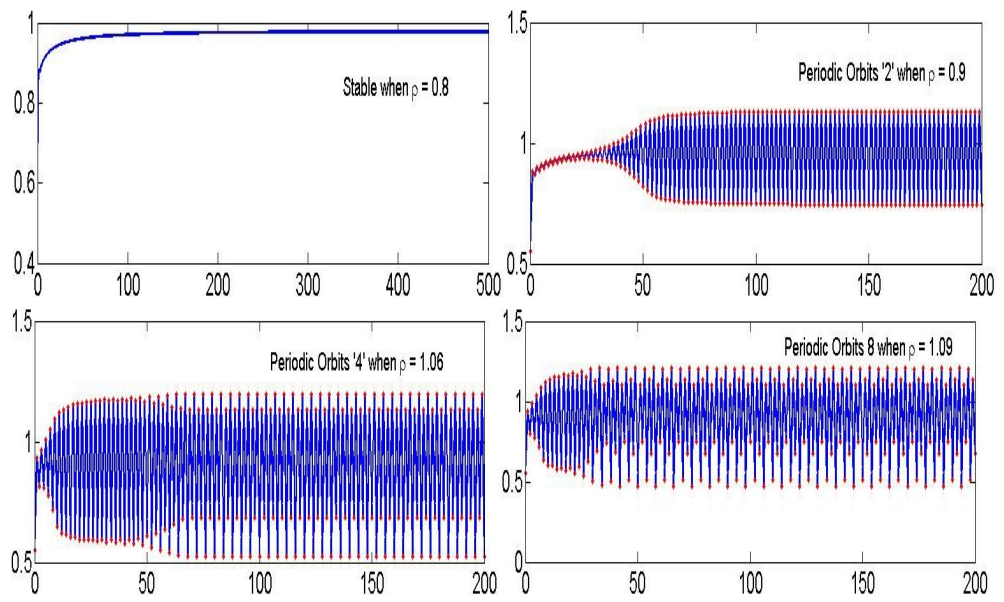

FiguRe 3. Different periodic orbits of the axial Bifurcation of the system (3)

Example 11. Taking $\nu=0.85, s=0.35, \beta=0.4, c=0.01, w=0.01, \eta=0.56, f=$ 0.12 and $2 \leq \rho \leq 4.5$ in system (3) and $x(0)=0.55, y(0)=0.45$, we get $E S_{2}=$ $\left(x^{*}, y^{*}\right)=(0.2321,0.6469)$. For $M=-0.0804 ; N=0.0336, S^{*}=-0.1279, \rho_{3}=$ 

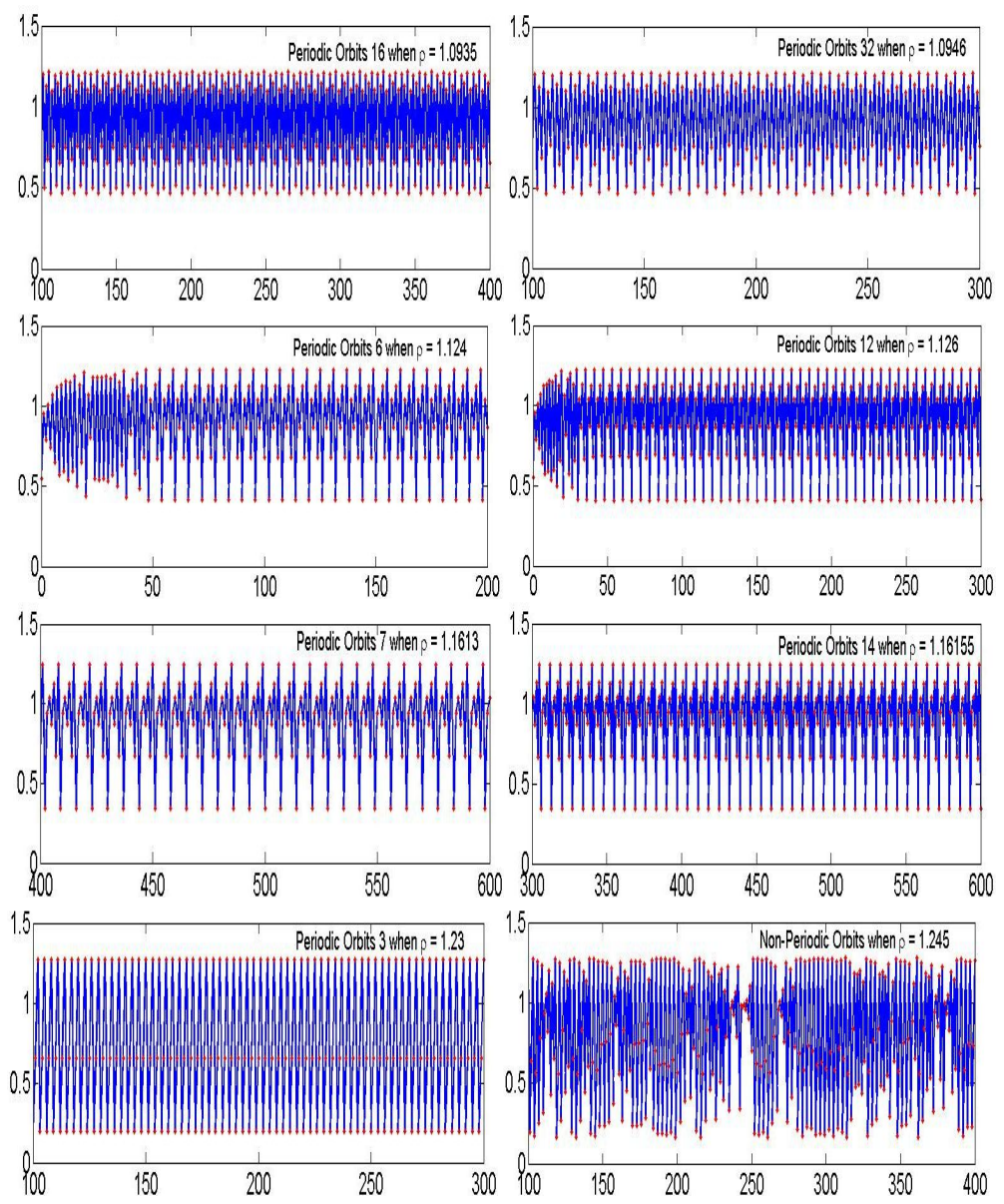

FiguRE 4. Different periodic orbits of the axial Bifurcation of the system (3)

2.6124 , the eigen values are $m_{1,2}=0.9038 \pm i 0.4280$ with $\left|\eta_{1,2}\right|=1$. The critical value given in Theorem (8) is $\rho_{3}=2.6124$.

The bifurcation diagrams in $x$ and $y$ plane are presented in Figure 5(a), 5(b). Phase trajectories obtained for various values of $\rho$ are given in Figure 6 and Figure 7. System (3) at $E S_{2}$ is locally asymptotic stable for $\rho<\rho_{3}=2.6124$ and becomes unstable at $\rho=\rho_{3}$ followed by formation of invariant cycles for $\rho>\rho_{3}$ which are presented in Figure 5, Figure 6 and Figure 7.

Example 12. Consider the values $\nu=0.85, \rho=2.67, s=0.35, \beta=0.4, c=$ $0.01, w=0.01, \eta=0.56, f=0.12$ and $x(0)=0.55, y(0)=0.45$. The occurrence 

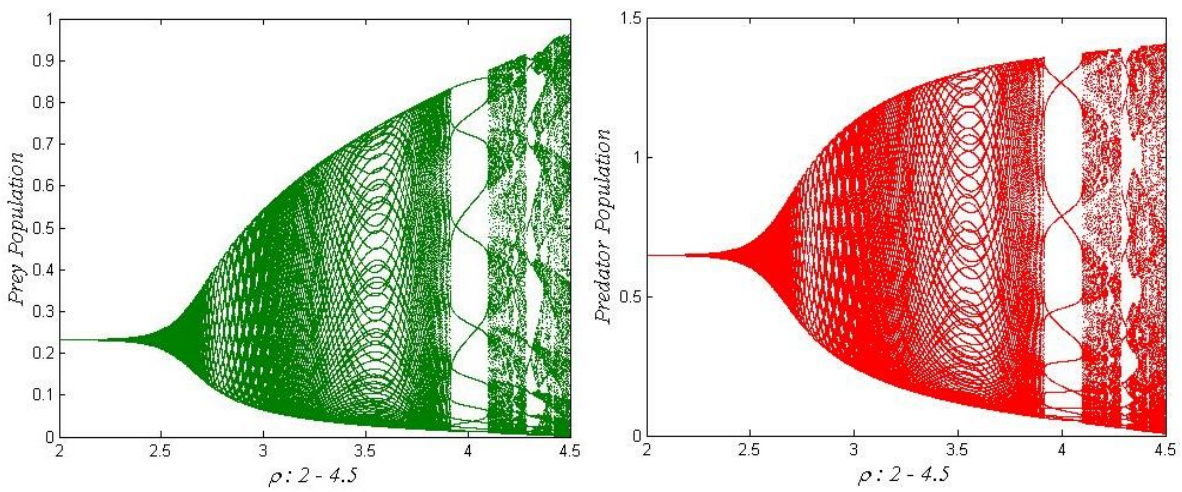

FiguRE 5. Neimark- Sacker Bifurcations for the system 3
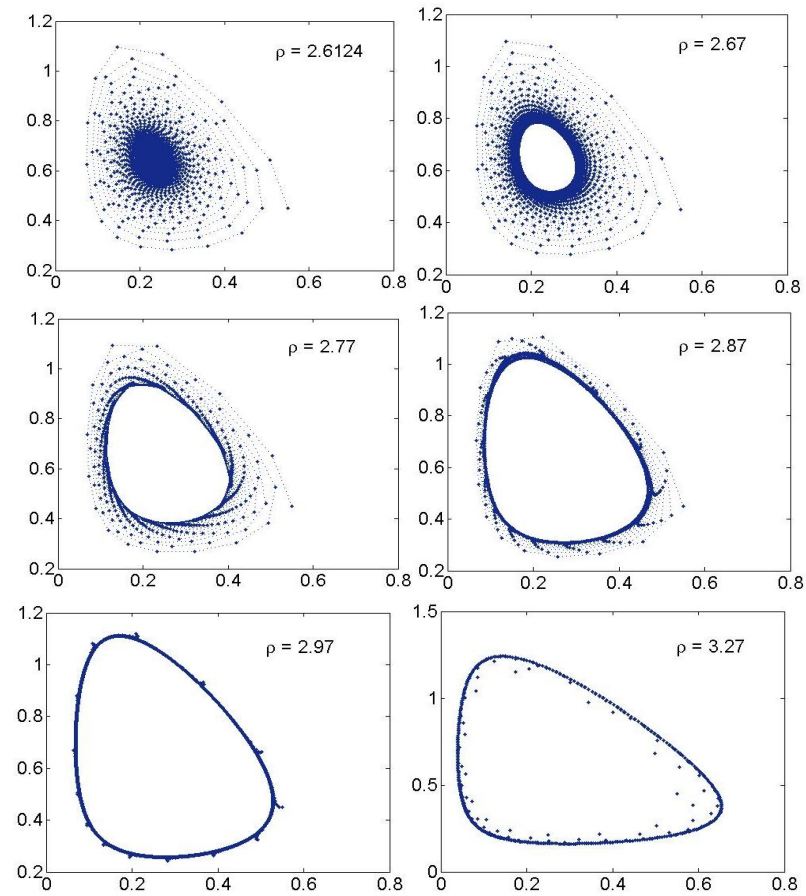

Figure 6. Phase portraits of system (3) for different values of $\rho$

of the Neimark-Sacker bifurcation for $\rho \in[2,4.5]$ of the system (3) is illustrated 

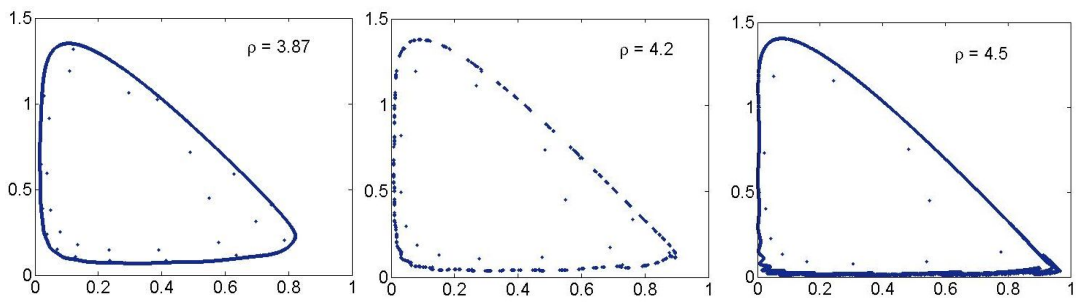

Figure 7. Phase portraits of system $(3)$ for different values of $\rho$

in Example (11). The unstable closed path formed at $\rho=2.67$ enclosing unstable steady state $E S_{2}=\left(x^{*}, y^{*}\right)=(0.2321,0.6469)$ is presented in Figure-8.
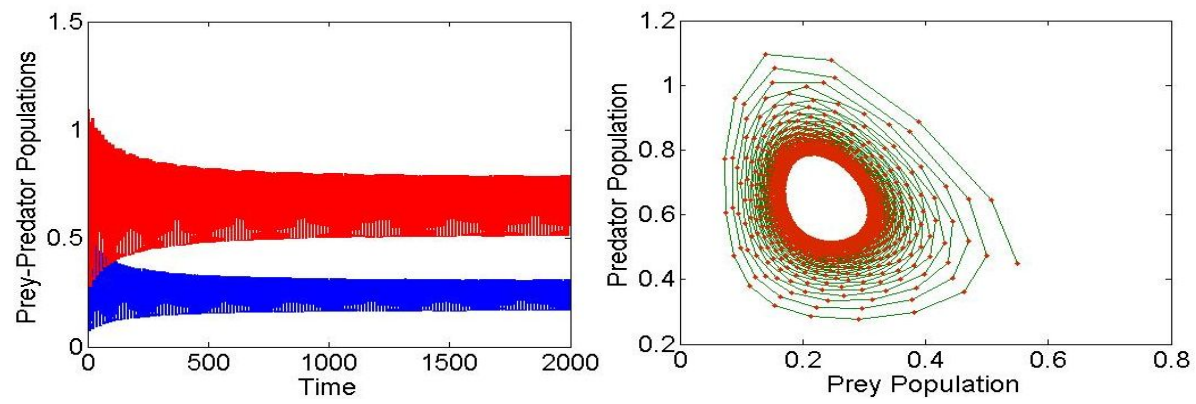

Figure 8. Phase portrait for the system 3 with time series plot

The controlled system (13) for above values can be written as

$$
\begin{aligned}
& x(t+1)=x(t)+\frac{\alpha \rho^{\nu}}{\Gamma(1+\nu)}[s x(t)(1-x(t))-\beta x(t) y(t)-c x(t)] \\
& y(t+1)=y(t)+\frac{\alpha \rho^{\nu}}{\Gamma(1+\nu)}[-w y(t)+\eta x(t) y(t)-f y(t)]
\end{aligned}
$$

with $\nu=0.85, \rho=2.67, s=0.35, \beta=0.4, c=0.01, w=0.01, \eta=0.56, f=0.12$ and $0<\alpha<1$. The stability of $E S_{2}$ is confirmed by the phase trajectory and time plots in Figure 9 for 16 at $\alpha=0.95$.

\section{Conclusion}

The qualitative study of the fractional order discrete equations of the prey- predator model with harvesting is carried out. The stability conditions and bifurcation analysis of the system is studied. The chaos control is performed with feedback control and numerical simulations for bifurcations with different phase trajectories are performed as well in accordance with the theoretical work. The periodic windows and different time plots are provided to understand the dynamics exhibited 

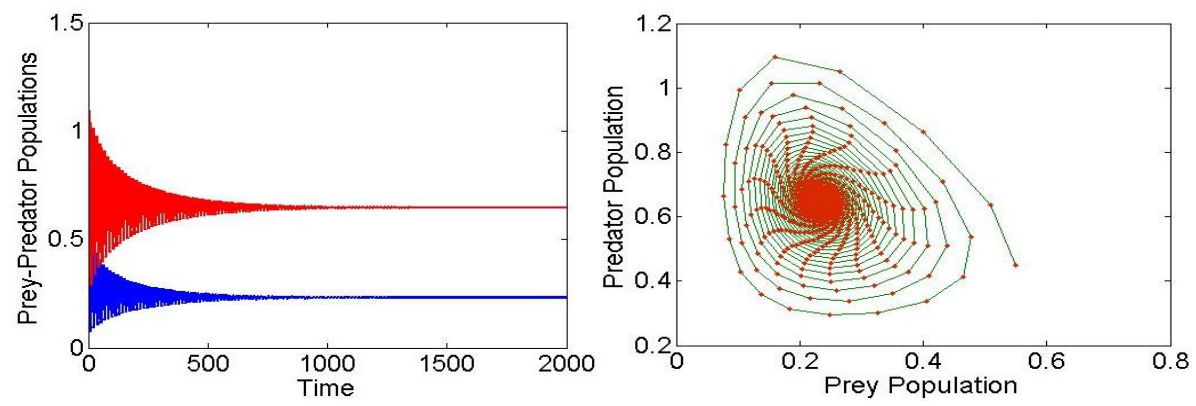

Figure 9. Phase portrait for the system 13 with time series plot

by the prey predator model.

Author Contribution Statements All authors jointly worked on the results and they read and approved the final manuscript.

Declaration of Competing Interests The authors declare that they have no competing interest.

\section{REFERENCES}

[1] Abdeljawad, T., Jarad, F., Alzabut, J., Fractional proportional differences with memory, The European Physical Journal Special Topics, 226 (16-18) (2017), 3333-3354, https://dx.doi.org/10.1140/epjst/e2018-00053-5.

[2] Agarwal, R. P., El-Sayed, A. M. A., Salman, S. M., Fractional order Chua's system: discretization, bifurcation and chaos, Adv. Difference Equ., 2013 (320) (2013), 1-13, https://dx.doi.org/10.1186/1687-1847-2013-320.

[3] Alzabut, J., Abdeljawad, T., A generalized discrete fractional Gronwall inequality and its application on the uniqueness of solutions for nonlinear delay fractional difference system, Applicable Analysis and Discrete Mathematics, 12 (1) (2018), 36-48, https://dx.doi.org/jstor.org/stable/90020603.

[4] Alzabut, J., Tyagi, S., Abbas, S., Discrete fractional-order BAM neural networks with leakage delay: Existence and stability results, Asian Journal of Control, 22 (1) (2018), 143-155, https://dx.doi.org/10.1002/asjc.1918.

[5] Caputo, M., Linear models of dissipation whose $\mathrm{q}$ is almost frequency independent, Geophys. J. R. Astr. Soc., 13 (5) (1967), 529-539, https://dx.doi.org/10.1111/j.1365246X.1967.tb02303.x.

[6] Din. Q,, Complexity and chaos control in a discrete-time prey-predator model, Communications in Nonlinear Science and Numerical Simulation, 49 (2017), 113-134, https://dx.doi.org/10.1016/j.cnsns.2017.01.025.

[7] El Raheem, Z. F., Salman, S. M., On a discretization process of fractionalorder logistic differential equation, J. Egypt. Math. Soc., 2 (2014), 407-412, https://dx.doi.org/10.1016/j.joems.2013.09.001. 
[8] El-Saka, H. A., Ahmed, E., Shehata, M. I., El-Sayed, A. M. A., On stability, persistence, and Hopf bifurcation in fractional order dynamical systems, Nonlinear Dyn., 56 (12) (2019), 121-126, https://dx.doi.org/10.1007/s11071-008-9383-x.

[9] Elaydi, S. N., An Introduction to Difference Equations, Springer-Verlag, New York, 2005.

[10] Elaydi, S. N., Discrete Chaos: with Applications in Science and Engineering, Chapman and Hall/CRC, Baca Raton, 2008.

[11] Elsadany, A. A., Matouk, A. E., Dynamical behaviors of fractional-order Lotka-Volterra predator-prey model and its discretization, J. Appl. Math. Comput., 49 (2015), 269-283, https://dx.doi.org/10.1007/s12190-014-0838-6.

[12] Gumus, O. A., Neimark-Sacker bifurcation and stability of a prey-predator system, Miskolc Mathematical Notes, 21 (2) (2020), 873-885, https://dx.doi.org/10.18514/MMN.2020.3386.

[13] Gumus, O. A., Selvam, A. G. M., Janagaraj, R., Stability of modified host-parasitoid model with Allee effect, Applications and Applied Mathematics: An International Journal, 15 (2) (2020), 1032-1045.

[14] Gumus, O. A., Yalcin, Y., Stability and Hopf bifurcation analysis of delay prey-predator model, Journal of Science and Arts, 20 (2) (2020), 277-282.

[15] Guo, Y., The stability of solutions for a fractional predator - prey system, Abstr. Appl. Anal., 2014 (2014), 124-145.

[16] Kar, T. K., Chaudhuri, K. S., On non-selective harvesting of two competing fish species in the presence of toxicity, Ecol. Model, 161 (2003), 125-137, https://dx.doi.org/10.1016/S03043800(02)00323-X.

[17] Li, H. L., Long, Z., Cheng, H., Yao-Lin, J., Zhidong, T., Dynamical analysis of a fractionalorder predator-prey model incorporating a prey refuge, J. Appl. Math. Comput., 54 (2016), 435-449, https://dx.doi.org/10.1007/s12190-016-1017-8.

[18] Liu, X., Xiao, D., Complex dynamic behaviors of a discrete-time predator - prey system, Chaos Solitons Fractals, 32 (2007), 80-94, https://dx.doi.org/10.1016/j.chaos.2005.10.081.

[19] Makinde, O. D., Solving ratio-dependent predator-prey system with constant effort harvesting using Adomian decomposition method, Appl. Math. Comput., 186 (2007), 17-22, https://dx.doi.org/10.1016/j.amc.2006.07.083

[20] Myerscough, M. R., Gray, B. F., Hogarth, W. L., Norbury, J., An analysis of an ordinary differential equation model for a two-species predator prey system with harvesting and stocking, J. Math. Biol., 30 (1992), 389-411, https://dx.doi.org/10.1007/BF00173294.

[21] Selvam, A. G. M., Dhineshbabu, R., Gumus, O. A., Complex dynamics behaviors of a discrete prey-predator-scavenger model with fractional order, Journal of Computational and Theoretical Nanoscience, 17 (5) (2020), 2139-2146, https://dx.doi.org/10.1166/jctn.2020.8860.

[22] Selvam, A. G. M., Dhineshbabu, R., Gumus, O. A., Stability and Neimark-Sacker bifurcation for a discrete system of one-scrool chaotic attractor with fractional order, IOP Conf. Series: Journal of Physics: Conf. Series, 1597 (012009) (2020), 1-10, https://dx.doi.org/10.1088/1742-6596/1597/1/012009.

[23] Selvam, A. G. M., Dhineshbabu, R., Jacob, S. B., Quadratic harvesting in a fractional order scavenger model, IOP Conf. Series: Journal of Physics: Conf. Series, 1139 (012002) (2018), 1-8, https://dx.doi.org/10.1088/1742-6596/1139/1/012002.

[24] Selvam, A. G. M., Janagaraj, R., Jacintha, M., Stability, bifurcation, chaos: discrete prey predator model with step size, International Journal of Innovative Technology and Exploring Engineering, 9 (1) (2019), 3382-3387, https://dx.doi.org/10.35940/ijitee.A4866.119119.

[25] Selvam, A. G. M., Janagaraj, R., Vignesh, D., Allee effect and Holling type - ii response in a discrete fractional order prey - predator model, IOP Conf. Series: Journal of Physics: Conf. Series, 1139 (012003) (2018), 1-7, https://dx.doi.org/10.1088/1742-6596/1139/1/012003.

[26] Sohel Rana, S. M., Bifurcation and complex dynamics of a discrete-time predator - prey system, Computational Ecology and Software, 5 (2) (2015), 187-200. 